\title{
BMJ Open Predictors of physical functioning after total hip arthroplasty: a systematic review
}

\author{
L D Buirs, ${ }^{1}$ L W A H Van Beers, ${ }^{1}$ V A B Scholtes, ${ }^{1}$ T Pastoors, ${ }^{1}$ S Sprague, ${ }^{2}$ \\ R W Poolman ${ }^{1}$
}

To cite: Buirs LD, Van Beers LWAH, Scholtes VAB, et al. Predictors of physical functioning after total hip arthroplasty: a systematic review. BMJ Open 2016;6: e010725. doi:10.1136/ bmjopen-2015-010725

- Prepublication history for this paper is available online. To view these files please visit the journal online (http://dx.doi.org/10.1136/ bmjopen-2015-010725).

Received 30 November 2015 Revised 30 May 2016 Accepted 1 June 2016

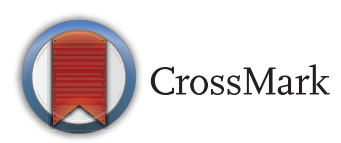

\footnotetext{
${ }^{1}$ Department of Orthopaedic Surgery, Onze Lieve Vrouwe Gasthuis (OLVG),

Amsterdam, The Netherlands ${ }^{2}$ Division of Orthopedic Surgery, Department of Surgery, Centre for EvidenceBased Orthopedics, McMaster University, Hamilton, Ontario, Canada
}

Correspondence to Dr LD Buirs; Idbuirs@gmail.com

\section{ABSTRACT}

Objective: The objective of this systematic review of the literature was to identify the predictors of functional outcome after total hip arthroplasty (THA).

Method: A systematic literature search in Web of Science, CINAHL, EMBASE and PubMed was conducted on 23 June 2015. The articles were selected based on their quality, relevance and measurement of the predictive factor. The level of evidence of all studies was determined using the GRADE rating scheme.

Results: The initial search resulted in 1092 citations. After application of the inclusion and exclusion criteria, 33 articles met our eligibility criteria and were graded. Included studies were classified as level of evidence low (11), moderate (17) or high (5). Of the included studies, 18 evaluated body mass index (BMI), 17 evaluated preoperative physical functions, 15 evaluated age, 15 evaluated gender and 13 evaluated comorbidity. There was strong evidence suggesting an association between BMI, age, comorbidity, preoperative physical functions and mental health with functional outcome after THA. There was weak evidence suggesting an association between quadriceps strength and education with functional outcome after THA. The evidence was inconsistent for associations with gender and socioeconomic status and functional outcome following THA. We found limited evidence suggesting that alcohol consumption, vitamin $D$ insufficiency and allergies were predictors of functional outcome following THA.

Conclusions: We have identified multiple predictors of functional outcome after THA, which will enable general practitioners and orthopaedic surgeons to better predict the improvement in physical functioning for their patients with THA. They can use this information to provide patient-specific advice regarding the referral for THA and the expected outcomes after THA. Further research with consistent measurement tools, outcomes and duration of follow-up across studies is needed to confirm the influence of these factors.

\section{INTRODUCTION}

Total hip arthroplasty (THA) is a surgical procedure performed to reduce pain and improve function in patients with osteoarthritis (OA) of the hip. According to the

\section{Strengths and limitations of this study}

- We have carried out a comprehensive and robust systematic review in accordance with the PRISMA guidelines.

- We included a range of patient-related predictors and did not limit ourselves to the most common predictors. This led to a broad overview of predictors evaluated.

- We screened a large number of literature sources, and all reviewing and data extraction was carried out by one author (LDB) and double checked by a second author (LWAHVB).

- Owing to the heterogeneity across studies regarding measurement tool, predictor and duration of follow-up we could not apply a meta-analysis.

- The predictors like quadriceps strength, education, socioeconomic status and alcohol consumption were reported only a few times and therefore conclusions cannot be reached.

Agency for Healthcare Research and Quality, more than 305000 total hip replacements are performed each year in the USA. ${ }^{1}$ Following THA, the majority of patients experience reductions in pain, improvements in function and better health-related quality of life. ${ }^{2}$ However, not all patients achieve the same level of functional improvement after THA. Specifically, more than $30 \%$ of patients undergoing THA report moderate-to-severe activity limitations 2 years post-THA. ${ }^{3}$ It is unclear which factors are associated with these limitations in function. ${ }^{45}$

In the previous decade, many studies have been published investigating the predictors of functional outcome after THA. Young et al published a systematic review on this topic in 1998. Since then considerable research has been published on predictors of functional outcome which justifies a new systematic review. ${ }^{6}$ Therefore, we conducted a systematic review of predictors of mid-term and longterm functional outcome after THA. 


\section{METHODS}

\section{Registration}

This systematic review is registered at Prospero (http:// www.crd.york.ac.uk/PROSPERO/) with registry number CRD42015016929.

\section{Selection criteria}

Studies that met the following criteria were included in our review: (1) included patients undergoing a THA; (2) included physical functioning was an outcome measure; (3) had at least one variable that was considered as a predictor of physical functioning and (4) was written in English. We did not select a time period.

\section{Search strategy}

With the guidance of an independent medical librarian, we conducted a literature search through four medical databases: Web of Science; CINAHL; EMBASE and PubMed. This literature search was performed on 23 June 2015. In Web of Science we used the following search terms: TOPIC: (total hip arthroplasty) AND TOPIC: (predictor*). In CINAHL we searched for: (MM "Arthroplasty, Replacement, Hip") AND predictor*. In EMBASE we searched for: exp hip arthroplasty/exp prediction/or exp predictor variable/exp prognosis/or exp functional assessment/or exp treatment outcome/or exp daily life activity/. In PubMed we searched for ("Arthroplasty, Replacement, Hip"(Majr) OR "Hip Prosthesis"(Majr)) AND (predictor* OR risk Factor* OR risk assessment OR predictive value of tests OR prognostic factor* OR Prognostic*) AND (HOOS OR "hip disability and osteoarthritis outcome score" OR WOMAC OR "Western Ontario and McMaster Universities Arthritis Index" OR "Harris hip score" OR HHS OR SF-12 OR short form 12 OR SF 36 OR "short form 36" OR Trendelenburg OR TUG OR "timed up and go" OR "Oxford hip score" OR "IOWA hip score" OR "Functional recovery score" OR FRS OR AFI OR "Hospital for special surgery" OR AAOS OR "Charnley hip score" OR HSS OR LEGS OR "Mayo clinical hip score"). The results of these four different searches were combined in Reference Manager and duplicates were discarded.

\section{Study selection}

Two of the authors (LWAHVB and TP) independently screened the titles and abstracts of all the articles using the aforementioned selection criteria. Both reviewers screened the full-text articles of the articles found eligible in the first round. A third author (LDB) compared these results and in case of different opinions, a consensus was reached. The study selection procedure is schematically presented in figure 1 .

\section{Data extraction}

One of the authors (LDB) extracted the data, which was double checked by a second author (LWAHVB). From each article, the following information was extracted: (1) predictor variable; (2) author; (3) year of publication; (4) level of evidence; (5) number of patients; (6) measurement tools used; (7) follow-up period; (8) significance level; (9) association between predictor variable and outcome measure; and (10) predictor level of measurement (table 1). The results were categorised by predictor variable.

\section{Methodological quality assessment}

The level of evidence of all studies was determined by one of the authors (LDB) using the GRADE rating scheme (http://www.gradeworkinggroup.org).

\section{Measurement tools}

We aimed to include all predictors mentioned in previous studies, and did not limit ourselves to the most common predictors. Some of the widely used measurement tools to define functional outcome are the Harris Hip Score (HHS), ${ }^{7}$ Oxford Hip Score (OHS), ${ }^{8}{ }^{9}$ Short Form-36 (SF-36) ${ }^{10}$ Lower Extremity Functional Scale (LEFS), ${ }^{11}$ Timed Up and Go (TUG) test ${ }^{12}{ }^{13}$ and the Western Ontario and McMaster Universities OA Index (WOMAC). ${ }^{14}$ We used all these measurement tools as outcome in this study.

\section{Best evidence synthesis}

A follow-up period up to 24 months was considered as 'short term' and a follow-up period of more than 24 months was considered as 'long term'. Results were divided into four categories of evidence: strong evidence: at least $60 \%$ of the studies, with a minimum of three studies, describing the same significant $(p<0.05)$ association. Weak evidence: (1) only two studies describe the same significant association; (2) three studies describe the same association out of which two are significant and one is not significant $(p>0.05)$. Limited evidence: (1) only one study available; (2) more studies were available of which none found a significant association. Inconsistent evidence: all other scenarios. ${ }^{15}$ No conclusions can be drawn in this literature review when no or inconsistent evidence is available.

This systematic review conforms to the PRISMA statement. $^{16}$

\section{RESULTS}

Selection and methodological quality

The initial search resulted in 1092 citations (figure 1) and 33 articles met our eligibility criteria. The articles included were designated as level of evidence low (11), moderate (17) or high (5; table 1 )

\section{Measures of functional outcome}

Multiple outcome measures were used across these studies including the HHS, OHS, SF-36 physical function (PF), LEFS, TUG and the WOMAC score. The follow-up period ranged from 3 to 72 months with an average of 18 (SD 17) months (table 2). 
Figure 1 Flow chart of the study selection procedure.
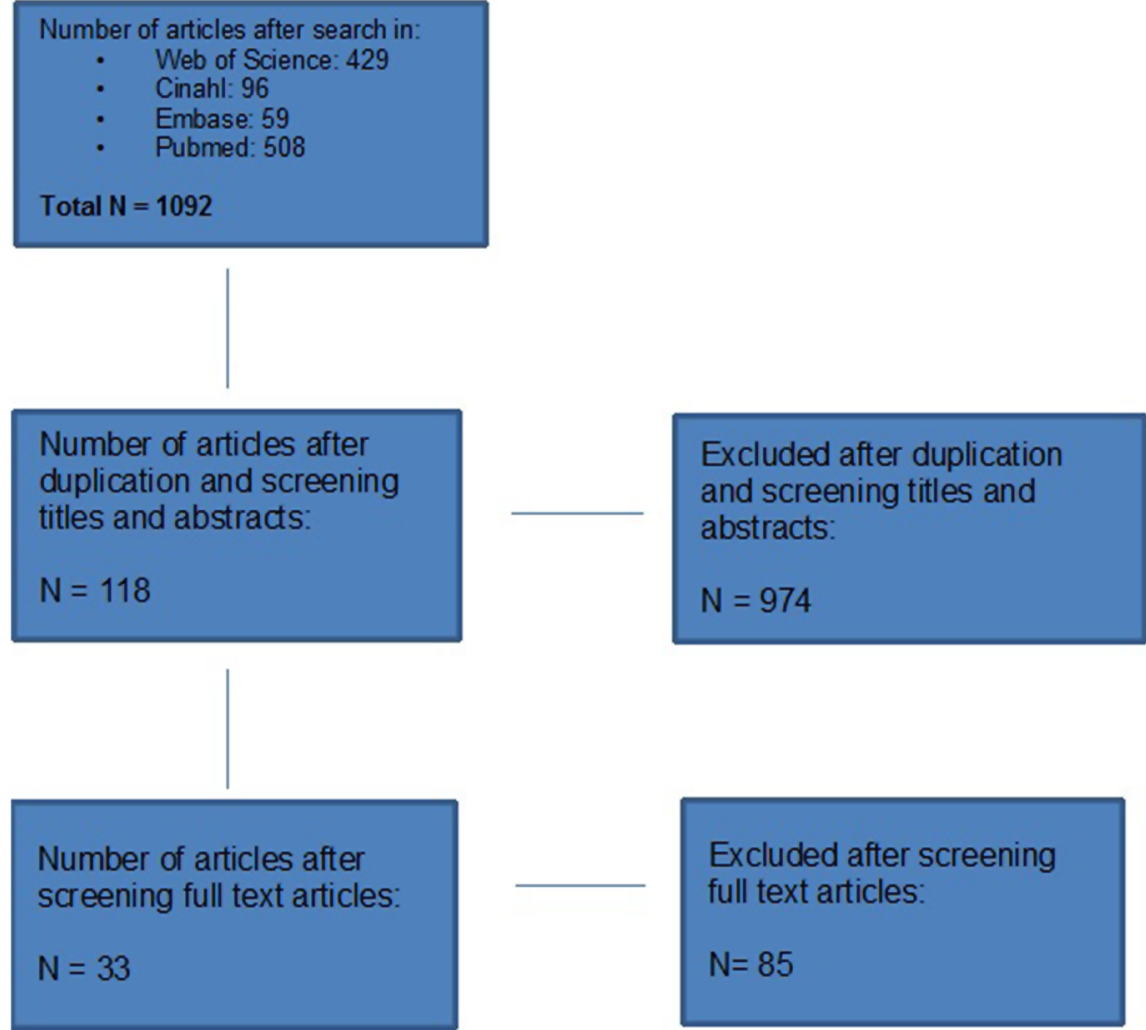

Excluded after screening full text articles:

$\mathrm{N}=85$
Predictive factors of functional outcome Body mass index

Eighteen studies evaluated body mass index (BMI) as a potential predictor of functional outcome after $\mathrm{THA}^{17-34}$ (table 3). A total of 14432 patients were included in all articles concerning the impact of BMI, with a mean follow-up time of 22 months. The applied levels of measurement of BMI were continuous, dichotomous or categorical.

The measurement tools used to determine the functional outcome were the WOMAC score, HHS, OHS, LEFS, SF-12 PF and the ambulatory status. The classification of a high BMI ranged from $>28$ to $>35 \mathrm{~kg} / \mathrm{m}^{2}$.

Of the 18 studies, 13 found a significant association. ${ }^{17-19}$

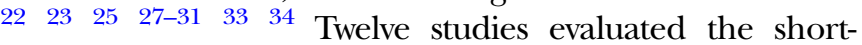
term functional outcome of which eight studies $^{17} 202225283033 \quad 34$ found a significant negative association and one article had a significant positive association. ${ }^{31}$ Of the seven studies evaluating the longterm functional outcome, five articles found a significant negative association. ${ }^{18} 192327{ }^{29}$ Studies were designated as level of evidence low (5), moderate (9) or high (4).

Since more than $60 \%$ of the studies report a significant negative association, there is strong evidence of a negative association between BMI and short-term and long-term functional outcomes after THA. These results were consistent when we only considered the studies with high or moderate levels of evidence according to GRADE.

Age

Fifteen studies evaluated age as a possible predictor of functional outcome after THA ${ }^{17} 18212324$ 26-30 32 34-37 (table 4). A total of 9234 patients were included in all studies that identified age as a possible predictor, with a mean follow-up time of 19 months. The applied levels of measurement of age were continuous, dichotomous or categorical.

The measurements tools used to determine the functional outcome were the WOMAC score, HHS, OHS, 
Table 1 Methodological quality of included studies

\begin{tabular}{|c|c|c|c|c|c|c|c|}
\hline Study & Study design & Risk of bias & Inconsistency & Indirectness & Imprecision & Other considerations & Grade \\
\hline Kessler and Käfer ${ }^{24}$ & Observational study & Not serious & Not serious & Not serious & Not serious & Strong association & Moderate \\
\hline Aranda Villalobos et $a^{\beta 1}$ & Observational study & Not serious & Not serious & Not serious & Not serious & None & Low \\
\hline Nankaku et $a^{P^{6}}$ & Observational study & Not serious & Not serious & Not serious & Not serious & Strong association & Moderate \\
\hline Slaven 28 & Observational study & Not serious & Not serious & Not serious & Not serious & None & Low \\
\hline Moran et $a^{R^{5}}$ & Observational study & NA & Not serious & Not serious & Not serious & Strong association & Moderate \\
\hline Stevens et $a^{\beta 0}$ & Observational study & Not serious & Not serious & Not serious & Not serious & Strong association & Moderate \\
\hline Wang et $a{ }^{\beta 2}$ & Observational study & Not serious & Not serious & Not serious & Not serious & None & Moderate \\
\hline Dowsey et $a^{\text {Po }}$ & Observational study & Serious & Not serious & Not serious & Not serious & Strong association & Low \\
\hline Judge et $a \beta^{\beta 3}$ & Observational study & Not serious & Not serious & Not serious & Not serious & Very strong association & High \\
\hline Bergschmidt et al ${ }^{17}$ & Observational study & Not serious & Not serious & Not serious & Not serious & Strong association & Moderate \\
\hline Jones et $a^{R^{2}}$ & Observational study & Not serious & Not serious & Not serious & Not serious & Strong association & Moderate \\
\hline Smith et $a R^{29}$ & Observational study & Not serious & Not serious & Serious & Not serious & Strong association & Moderate \\
\hline Judge et $a P^{3}$ & Observational study & Not serious & Not serious & Not serious & Not serious & Very strong association & High \\
\hline Bischoff et al $1^{18}$ & Observational study & Not serious & Not serious & Not serious & Not serious & Strong association & Moderate \\
\hline Gandhi et $a^{f^{1}}$ & Observational study & Serious & Not serious & Not serious & Not serious & None & Low \\
\hline Nilsdotter et $a^{R^{7}}$ & Observational study & Not serious & Serious & Not serious & Not serious & Strong association & Low \\
\hline Davis et al ${ }^{19}$ & Observational study & Not serious & Not serious & Not serious & Not serious & Very strong association & High \\
\hline Hamilton et $a l^{\beta 5}$ & Observational study & Not serious & Not serious & Not serious & Not serious & None & Low \\
\hline Quintana et $a \beta^{\beta 7}$ & Observational study & Not serious & Not serious & Not serious & Not serious & Strong association & Moderate \\
\hline Nilsdotter and Lohmander ${ }^{36}$ & Observational study & Not serious & Not serious & Not serious & Not serious & Strong association & Moderate \\
\hline Dowsey et $a{ }^{34}$ & Observational study & Not serious & Not serious & Not serious & Not serious & Very strong association & High \\
\hline Lavernia, $2011^{38}$ & Observational study & Serious & Not serious & Not serious & Not serious & Strong association & Low \\
\hline Mahomed et a ${ }^{\beta 9}$ & Observational study & Not serious & Not serious & Not serious & Not serious & Strong association & Moderate \\
\hline Vogl et $a f^{43}$ & Observational study & Not serious & Serious & Not serious & Not serious & NA & Low \\
\hline Clement et $a f^{42}$ & Observational study & Not serious & Not serious & Not serious & Not serious & Very strong association & High \\
\hline Johansson et $a l^{40}$ & Observational study & Not serious & Not serious & Not serious & Not serious & Strong association & Moderate \\
\hline Fortin et a ${ }^{41}$ & Observational study & Not serious & Not serious & Not serious & Serious & Strong association & Low \\
\hline Badura-Brzoza et al ${ }^{44}$ & Observational study & Not serious & Not serious & Not serious & Not serious & Strong association & Moderate \\
\hline Holstege et $a f^{46}$ & Observational study & Not serious & Not serious & Not serious & Not serious & Strong association & Moderate \\
\hline Schafer et $a l^{47}$ & Observational study & Not serious & Not serious & Not serious & NA & Strong association & Low \\
\hline Graves et a/ ${ }^{48}$ & Observational study & Not serious & Not serious & Not serious & Not serious & Strong association & Moderate \\
\hline Lavernia, $2014^{49}$ & Observational study & Not serious & Not serious & Not serious & NA & None & Low \\
\hline Lavernia et $a{ }^{45}$ & Observational study & Not serious & Not serious & Not serious & Not serious & Strong association & Moderate \\
\hline \multicolumn{8}{|c|}{$\begin{array}{l}\text { High: true effect lies close to the estimate of the effect. } \\
\text { Moderate: true effect is likely to be close to the estimate of the effect, but there is a possibility that it is substantially different. } \\
\text { Low: true effect may be substantially different from the estimate of effect. } \\
\text { Very low: true effect is likely to be substantially different from the estimate of effect. }\end{array}$} \\
\hline
\end{tabular}


Table 2 Characteristics of all included studies

\begin{tabular}{|c|c|c|c|c|c|c|}
\hline Author, year, nr & Age baseline & $\mathrm{N}$ of pts & Female (n, \%) & Inclusion criteria & Follow-up time & Measurement tool \\
\hline Badura-Brzoza, 2009, 42 & $61(54-75)$ & 156 & $59(58 \%)$ & Primary THA, OA & 6 months & SF-36 PF \\
\hline Bergschmidt, 2010, 113 & $66(58-74)$ & 100 & $48(50 \%)$ & Primary THA, OA & $6-12-24$ months & $\begin{array}{l}\text { HHS } \\
\text { WOMAC } \\
\text { SF-12 }\end{array}$ \\
\hline Bischoff, 2004, 51 & $73.1(65-93)$ & 922 & $60 \%$ & $\begin{array}{l}\text { OA, primary THA } \\
>65 \text { years }\end{array}$ & 3 years & WOMAC PF \\
\hline Clement, 2011, 101 & $68.1(65-74)$ & 1312 & NA & Primary OA, THR & 12 months & $\begin{array}{l}\text { OHS } \\
\text { SF-12 }\end{array}$ \\
\hline Davis, 2011, 100 & $69(34-96)$ & 1617 & 994 & Cemented THA & 5 years & $\begin{array}{l}\text { HHS } \\
\text { SF-36 PF }\end{array}$ \\
\hline Dowsey, 2010, 32 & 68.6/67/65.6 & 471 & $60.70 \%$ & Primary THA OA & 12 months & $\begin{array}{l}\text { HHS } \\
\text { SF-12 PF }\end{array}$ \\
\hline Dowsey, 2014, 15 & 68.4 & 835 & $60.10 \%$ & Primary THA & 12 months & SF-12 \\
\hline Fortin, 2002, 145 & 65.7 & 222 & $59 \%$ & Primary THA OA & 2 years & $\begin{array}{l}\text { WOMAC } \\
\text { SF-36 }\end{array}$ \\
\hline Hamilton, 2012, 17 & 68.1 & 1410 & $57.20 \%$ & Primary THA OA & $6-12$ months & $\begin{array}{l}\text { OHS } \\
\text { SF-12 }\end{array}$ \\
\hline Gandhi, 2010, 30 & $63.2(13.7)$ & 636 & $53.50 \%$ & $\begin{array}{l}<18 \text { years, } \\
\text { primary OA }\end{array}$ & 3.3 years & $\begin{array}{l}\text { WOMAC } \\
\text { SF-36 PF }\end{array}$ \\
\hline Graves, 2014, 29 & 59.5 & 459 & $61.00 \%$ & THA OA & 10. 4 months & $\begin{array}{l}\text { WOMAC } \\
\text { SF-36 }\end{array}$ \\
\hline Holstege, 2011, 102 & $72.7(6.8)$ & 55 & $41(74,5)$ & THA OA & 3 months & WOMAC PF \\
\hline Johansson, 2010, 114 & $67(7)$ & 75 & $36(48 \%)$ & THA OA & 6-12-24 months & $\begin{array}{l}\text { HHS } \\
\text { WOMAC } \\
\text { SF-36 }\end{array}$ \\
\hline Jones, 2012, 90 & $68.2(10.9)$ & 231 & $138(60 \%)$ & Primary THA & 6-36 months & WOMAC \\
\hline Judge, 2013, 14 & 70 & 1431 & $887(62 \%)$ & $\mathrm{OA}$ & $1-6$ years & $\mathrm{OHS}$ \\
\hline Kessler, 2007, nr 131 & 63.6 & 76 & $44.8(59 \%)$ & THA OA & 3 months & WOMAC \\
\hline Lavernia, 2014, 73 & 70 & 60 & $48(80 \%)$ & Primary THA & 3-24 months & $\begin{array}{l}\text { QWB-7 } \\
\text { SF-36 PF } \\
\text { WOMAC } \\
\text { HHS }\end{array}$ \\
\hline Lavernia, 2013, 81 & 62 & 191 & 70 & Primary THA & 12 months & $\begin{array}{l}\text { WOMAC } \\
\text { SF-36 }\end{array}$ \\
\hline Lavernia, 2011, 103 & $61(15)$ & 532 & $59 \%$ & THA & $6-7$ years & $\begin{array}{l}\text { SF-26 } \\
\text { HHS } \\
\text { WOMAC }\end{array}$ \\
\hline Mahomed, 2002, 149 & $66(9)$ & 103 & $57(55 \%)$ & THA OA & 6 months & $\begin{array}{l}\text { WOMAC PF } \\
\text { SF-36 PCS }\end{array}$ \\
\hline Moran, 2005, 136 & 68 & 749 & $61 \%$ & Primary THA & 6,18 months & HHS \\
\hline Nankaku, 2013, 83 & 60.4 & 204 & 173 & THA OA & 6 months & Ambulatory status \\
\hline Nilsdotter, 2002, 147 & 71 & 148 & 83 & THA OA & $3-6-12$ months & $\begin{array}{l}\text { WOMAC } \\
\text { SF-36 }\end{array}$ \\
\hline Nilsdotter, 2003, 52 & 71 & 211 & 106 & Primary THA & 3,6 years & WOMAC PF \\
\hline Quintana, 2009, 35 & 69.1 & 788 & $381(48 \%)$ & Primary THA OA & 6-24 months & $\begin{array}{l}\text { SF-36 PF } \\
\text { WOMAC }\end{array}$ \\
\hline Schafer, 2010, 110 & 61 & 1007 & $55 \%$ & Primary THA & 6 months & WOMAC \\
\hline Slaven, 2012, 15 & $68.2(8.2)$ & 40 & $22(55 \%)$ & Primary THA & 6 months & LEFS \\
\hline Smith, 2012, 92 & $68.5(9.9)$ & 1683 & NA & Primary THA & 3 years & $\mathrm{HHS}$ \\
\hline Stevens, 2012, 22 & $70.3(8.2)$ & 653 & $74.20 \%$ & Primary THA, OA & 52. 4 weeks & WOMAC \\
\hline Villalobos, 2012, 80 & $62.39(13.6)$ & 63 & $35(55.55 \%)$ & Primary THA & 3 months & $\begin{array}{l}\text { HHS } \\
\text { OHS } \\
\text { WOMAC } \\
\text { SF-12 PF }\end{array}$ \\
\hline Vogle, 2014, 108 & 68 & 321 & $58 \%$ & Primary THA & 6 months & WOMAC \\
\hline Wang, 2010, 107 & 61.65 & 97 & $62.40 \%$ & OA/osteonecrosis & 3-12-24 months & WOMAC \\
\hline
\end{tabular}

HHS, Harris Hip Score; LEFS, Lower Extremity Functional Scale; N of pts, number of patients; NA, not applicable; OHS, Oxford Hip Score; PCS, physical component summary scale; PF, physical function; QWB; quality of well-being index; SF-36 PF, Short Form 36 physical function; THA, total hip arthroplasty; THR, total hip replacement; WOMAC, Western Ontario and Mcmaster Universities Osteoarthritis Index. 
Table 3 Studies reporting BMI as possible predictor of functional outcome after THA

\begin{tabular}{|c|c|c|c|c|c|c|c|}
\hline Author, year & Grade & $\mathrm{N}$ of pts & $\begin{array}{l}\text { Measurement } \\
\text { tool }\end{array}$ & $\begin{array}{l}\text { FU period } \\
\text { (months) }\end{array}$ & $\begin{array}{l}\text { Significance } \\
\text { Level ( } p \text { value) }\end{array}$ & Association & Predictor level of measurement \\
\hline Kessler, 2007 & Moderate & 76 & WOMAC & ST $(3 \mathrm{~m})$ & 0.49 & No & Cont (BMI) \\
\hline \multirow{4}{*}{ Villalobos, 2012} & \multirow{4}{*}{ Low } & \multirow[t]{4}{*}{63} & SF-12 PCS & \multirow{4}{*}{$\mathrm{ST}(3 \mathrm{~m})$} & $0.004^{\star}$ & Pos & \multirow{4}{*}{$\begin{array}{l}\text { Dich } \\
\text { (1: BMl>28 2: BMI ?28) }\end{array}$} \\
\hline & & & WOMAC & & $0.041^{*}$ & Pos & \\
\hline & & & HHS & & $0.793^{\star}$ & No & \\
\hline & & & $\mathrm{OHS}$ & & $0.428^{*}$ & No & \\
\hline Nankaku, 2013 & Moderate & 204 & \multicolumn{2}{|c|}{ Ambulatory status ST (6m) } & 0.06 & No & Cont (BMI) \\
\hline Slaven, 2012 & Low & 40 & LEFS & $\mathrm{ST}(6 \mathrm{~m})$ & NA & Neg & $\begin{array}{l}\text { Dich } \\
\text { (1: BMl>34 2: BMI ?34) }\end{array}$ \\
\hline \multirow[t]{2}{*}{ Moran, 2005} & \multirow[t]{2}{*}{ Moderate } & \multirow[t]{2}{*}{749} & \multirow[t]{2}{*}{ HHS } & $\mathrm{ST}(6 \mathrm{~m})$ & 0.02 & Neg & \multirow[t]{2}{*}{ Cont (BMI) } \\
\hline & & & & ST $(18 \mathrm{~m})$ & 0.001 & Neg & \\
\hline Stevens, 2012 & Moderate & 653 & WOMAC & $\mathrm{ST}(12 \mathrm{~m})$ & 0.001 & Neg & Cont (BMI) \\
\hline Wang, 2010 & Moderate & 97 & WOMAC & ST $(12 \mathrm{~m})$ & 0.11 & No & Cont (BMI) \\
\hline \multirow[t]{2}{*}{ Dowsey, 2010} & \multirow[t]{2}{*}{ Low } & \multirow[t]{2}{*}{471} & HHS & \multirow[t]{2}{*}{$\mathrm{ST}(12 \mathrm{~m})$} & $<0.01$ & Neg & \multirow{2}{*}{$\begin{array}{l}\text { Cat (3) } \\
(1: \mathrm{BMl}<30 \text { 2: } \mathrm{BMl} 30-39 \text { 3: } \mathrm{BMl} \geq 40)\end{array}$} \\
\hline & & & SF-12 PCS & & 0.05 & Neg & \\
\hline Dowsey, 2014 & High & 835 & HHS & ST $(12 \mathrm{~m})$ & $<0.0001$ & Neg & Cont (BMI) \\
\hline Judge, 2014 & High & 4413 & $\mathrm{OHS}$ & ST (12 m) & 0.003 & Neg & $\begin{array}{l}\text { Cat (5) } \\
\text { (1: BMI 18.5-25 2: BMI 25-30 3: BMI 30-35 } \\
\text { 4: BMI 35-40 5: BMl>40) }\end{array}$ \\
\hline Bergschmidt, 2010 & Moderate & 100 & HHS & ST $(24 \mathrm{~m})$ & 0.007 & Neg & $\begin{array}{l}\text { Cat }(3) \\
(1: \mathrm{BMl}<26 \text { 2: } \mathrm{BMI} 26-29 \text { 3: } \mathrm{BMl}>29)\end{array}$ \\
\hline \multirow[t]{2}{*}{ Jones, 2012} & \multirow[t]{2}{*}{ Moderate } & \multirow[t]{2}{*}{231} & \multirow[t]{2}{*}{ WOMAC } & $\mathrm{ST}(6 \mathrm{~m})$ & 0.001 & Neg & Dich \\
\hline & & & & LT $(36 \mathrm{~m})$ & No & No & (1: BMI>35 2: BMI ?35) \\
\hline Smith, 2012 & Moderate & 1683 & HHS & LT $(36 \mathrm{~m})$ & $<0.01$ & Neg & Cont (BMI) \\
\hline Judge, 2013 & High & 1431 & $\mathrm{OHS}$ & LT (36 m) & $<0.001$ & $\mathrm{Neg}$ & Cont (BMI) \\
\hline Bischoff, 2004 & Moderate & 922 & WOMAC PF & LT (36 m) & NA & Neg & Cont (BMI) \\
\hline Gandhi, 2010 & Low & 636 & WOMAC & LT (39 m) & 0.06 & No & Cont (BMI) \\
\hline Nilsdotter, 2003 & Low & 211 & WOMAC PF & LT (42 m) & 0.03 & Neg & Cont (BMI) \\
\hline Davis, 2011 & High & 1617 & HHS & LT $(60 \mathrm{~m})$ & $<0.001$ & $\mathrm{Neg}$ & Cont (BMI) \\
\hline
\end{tabular}


Table 4 Studies reporting age as possible predictor of functional outcome after THA

\begin{tabular}{|c|c|c|c|c|c|c|c|}
\hline Author, year & Grade & $\mathrm{N}$ of pts & $\begin{array}{l}\text { Measurement } \\
\text { tool }\end{array}$ & $\begin{array}{l}\text { FU period } \\
\text { (months) }\end{array}$ & $\begin{array}{l}\text { Significance } \\
\text { Level ( } p \text { value) }\end{array}$ & Association & Predictor level of measurement \\
\hline Kessler, 2007 & Moderate & 76 & WOMAC & $\mathrm{ST}(3 \mathrm{~m})$ & 0.03 & Neg & Cont (age) \\
\hline Nankaku, 2013 & Moderate & 204 & \multicolumn{2}{|c|}{ Ambulatory status ST $(6 \mathrm{~m})$} & Yes & Neg & $\begin{array}{l}\text { Dich } \\
\text { (1: age >67.5 2: age ?67.5) }\end{array}$ \\
\hline Slaven, 2012 & Low & 40 & LEFS & $\mathrm{ST}(6 \mathrm{~m})$ & No & No & $\begin{array}{l}\text { Dich } \\
\text { (1: age >68.5 2: age ?68.5) }\end{array}$ \\
\hline Hamilton, 2012 & Low & 1410 & $\begin{array}{l}\text { OHS } \\
\text { SF-12 }\end{array}$ & $\begin{array}{l}\text { ST }(6 \mathrm{~m}) \\
\mathrm{ST}(12 \mathrm{~m})\end{array}$ & $\begin{array}{l}x \\
x\end{array}$ & $\begin{array}{l}\text { No } \\
\text { No }\end{array}$ & Cont (age) \\
\hline Quintana, 2009 & Moderate & 788 & WOMAC PF & $\begin{array}{l}\text { ST }(6 \mathrm{~m}) \\
\text { ST }(24 \mathrm{~m})\end{array}$ & $\begin{array}{l}0.41 \\
0.001\end{array}$ & $\begin{array}{l}\text { No } \\
\text { Neg }\end{array}$ & $\begin{array}{l}\text { Dich } \\
\text { (1: age >70 2: age ?70) }\end{array}$ \\
\hline Stevens, 2012 & Moderate & 653 & WOMAC & $\mathrm{ST}(12 \mathrm{~m})$ & 0.01 & $\mathrm{Neg}$ & Cont (age) \\
\hline Wang, 2010 & Moderate & 97 & WOMAC & $\mathrm{ST}(12 \mathrm{~m})$ & No & No & Cont (age) \\
\hline Dowsey, 2014 & High & 835 & $\begin{array}{l}\text { HHS } \\
\text { SF-12 PCS }\end{array}$ & ST (12 m) & $\begin{array}{l}<0.0001 \\
0.003\end{array}$ & $\begin{array}{l}\text { Neg } \\
\text { Neg }\end{array}$ & Cont (age) \\
\hline Nilsdotter, 2002 & Moderate & 148 & $\begin{array}{l}\text { WOMAC PF } \\
\text { SF-36 }\end{array}$ & ST $(12 \mathrm{~m})$ & $\begin{array}{l}0.004 \\
0.002\end{array}$ & $\begin{array}{l}\text { Neg } \\
\text { Neg }\end{array}$ & $\begin{array}{l}\text { Dich } \\
\text { (1: age >72 2: age ?72) }\end{array}$ \\
\hline $\begin{array}{l}\text { Bergschmidt, } \\
2010\end{array}$ & Moderate & 100 & $\mathrm{HHS}$ & ST (12 m) & $>0.097$ & No & Cat (3) \\
\hline & & & $\begin{array}{l}\text { WOMAC } \\
\text { SF-12 }\end{array}$ & & $\begin{array}{l}>0.097 \\
>0.097\end{array}$ & $\begin{array}{l}\text { No } \\
\text { No }\end{array}$ & (1: age $<60$ 2: age 60-69 3: age >69 \\
\hline Bischoff, 2004 & Moderate & 922 & WOMAC PF & $\mathrm{LT}(36 \mathrm{~m})$ & $\mathrm{x}$ & No & $\begin{array}{l}\text { Dich } \\
\text { (1: age >75 2: age ?75) }\end{array}$ \\
\hline Judge, 2013 & High & 1431 & $\mathrm{OHS}$ & $\mathrm{LT}(36 \mathrm{~m})$ & NA & Neg & $\begin{array}{l}\text { Cat (3) } \\
\text { (1: age <50 2: age } 50-60 \text { 3: age >60 }\end{array}$ \\
\hline Smith, 2012 & Moderate & 1683 & HHS & LT (36 m) & $<0.001$ & Neg & Cont (age) \\
\hline Nilsdotter, 2003 & Low & 211 & WOMAC PF & LT (43 m) & 0.002 & Neg & Cont (age) \\
\hline Gandhi, 2010 & Low & 636 & $\begin{array}{l}\text { WOMAC } \\
\text { SF-36 }\end{array}$ & LT (39 m) & $\begin{array}{l}<0.05 \\
<0.05\end{array}$ & Neg & Cont (age) \\
\hline $\begin{array}{l}\text { All significant resul } \\
\text { BMI, body mass in } \\
\text { patients; NA, not al } \\
\text { Ontario and Mcma }\end{array}$ & d.. & Ind & core; Pos, pos & $-36 \mathrm{PF}, \mathrm{SI}$ & 36 physical fun & $\begin{array}{l}\text { Extremity Fu } \\
\text { short-term; }\end{array}$ & $\begin{array}{l}\text { II Scale; LT, long-term; } N \text { of pts, number of } \\
\text { tal hip arthroplasty; WOMAC, Western }\end{array}$ \\
\hline
\end{tabular}


SF-36 PF, SF-12 PF and the ambulatory status. Different classifications of greater age were used, ranging from $>60$ to $>75$ years.

Of the 15 studies, 10 found a significant association. ${ }^{21} 232426272930343637$ Ten studies evaluated the short-term functional outcome of which six studies found a significant negative association. ${ }^{24} \begin{array}{llllll}26 & 30 & 34 & 36 & 37 & \text { The }\end{array}$ other four studies did not find a significant association. Of the six studies evaluating the long-term functional outcome, five studies found a significant negative association. ${ }^{21} 232936 \quad 37$ Studies were designated as level of evidence low (4), moderate (9) or high (2).

Since more than $60 \%$ of the studies report a significant negative association, there is strong evidence of a negative association between high age and short-term and long-term functional outcomes after THA. These results were consistent when we only considered the studies with high or moderate levels of evidence according to GRADE.
Gender

Fifteen studies evaluated gender as a possible predictor of functional outcome after $\mathrm{THA}^{17} \begin{array}{lllll}18 & 21 & 24 & 26-30\end{array}$ $32 \quad 34$ 36-38 (table 5). A total of 7156 patients were included in all studies that evaluated gender as a possible predictor, with a mean follow-up time of 23.3 months. The measurement tools used to determine the functional outcome included the WOMAC score, HHS, LEFS, SF-36 and the ambulatory status.

Of the 15 studies, 7 found a statistically significant association between preoperative physical function and functional outcome. ${ }^{21}$ 28-30 $32 \quad 3738$ Nine studies evaluated the short-term functional outcome of which four studies found a significant association. ${ }^{28} \quad 30 \quad 32 \quad 37$ Six studies evaluated the long-term functional outcome of which three found a significant association. ${ }^{21} 2938$ All studies were designated as level of evidence low (5), moderate (9) or high (1).

In four of the seven studies with a significant outcome, being male predicted a better outcome 29303237 whereas

Table 5 Studies reporting gender as possible predictor of functional outcome after THA

\section{Measurement FU period Significance}

\begin{tabular}{|c|c|c|c|c|c|c|c|}
\hline Author, year & Grade & $\mathrm{N}$ of pts & tool & (months) & Level ( $p$ value) & Association & $\begin{array}{l}\text { Predictor level of } \\
\text { measurement }\end{array}$ \\
\hline Kessler, 2007 & Moderate & 76 & WOMAC & ST (3 m) & NA & No & $\begin{array}{l}\text { Dich } \\
\text { (1: men 2: woman) }\end{array}$ \\
\hline Nilsdotter, 2002 & Moderate & 148 & $\begin{array}{l}\text { WOMAC } \\
\text { SF-36 }\end{array}$ & $\begin{array}{l}\text { ST }(3 \mathrm{~m}) \\
\text { ST }(12 \mathrm{~m})\end{array}$ & 0.7 & No & $\begin{array}{l}\text { Dich } \\
\text { (1: men 2: woman) }\end{array}$ \\
\hline Nankaku, 2013 & Moderate & 204 & \multicolumn{2}{|c|}{ Ambulatory status ST (6 m) } & 0.10 & No & $\begin{array}{l}\text { Dich } \\
\text { (1: men 2: woman) }\end{array}$ \\
\hline Slaven, 2012 & Low & 40 & LEFS & ST $(6 \mathrm{~m})$ & 0.039 & Pos, woman & $\begin{array}{l}\text { Dich } \\
\text { (1: men 2: woman) }\end{array}$ \\
\hline Quintana, 2009 & Moderate & 788 & SF-36 PF & $\begin{array}{l}\text { ST }(6 \mathrm{~m}) \\
\text { ST }(24 \mathrm{~m})\end{array}$ & $\begin{array}{l}\text { NA } \\
\text { NA }\end{array}$ & $\begin{array}{l}\text { Pos, men } \\
\text { No }\end{array}$ & $\begin{array}{l}\text { Dich } \\
\text { (1: men 2: woman) }\end{array}$ \\
\hline Bergschmidt, 2010 & Moderate & 100 & HHS & ST $(12 \mathrm{~m})$ & NA & No & $\begin{array}{l}\text { Dich } \\
\text { (1: men 2: woman) }\end{array}$ \\
\hline Stevens, 2012 & Low & 653 & WOMAC & ST $(12 \mathrm{~m})$ & 0.002 & Pos, men & $\begin{array}{l}\text { Dich } \\
\text { (1: men 2: woman) }\end{array}$ \\
\hline Dowsey, 2014 & High & 835 & HHS & $\mathrm{ST}(12 \mathrm{~m})$ & 0.06 & No & $\begin{array}{l}\text { Dich } \\
\text { (1: men 2: woman) }\end{array}$ \\
\hline Wang, 2010 & Moderate & 97 & WOMAC & ST (16.8 m) & 0.0001 & Pos, men & $\begin{array}{l}\text { Dich } \\
\text { (1: men 2: woman) }\end{array}$ \\
\hline Bischoff, 2004 & Moderate & 922 & WOMAC PF & LT (36 m) & No & No & $\begin{array}{l}\text { Dich } \\
\text { (1: men 2: woman) }\end{array}$ \\
\hline Jones, 2012 & Moderate & 231 & WOMAC & LT (36 m) & 0.118 & No & $\begin{array}{l}\text { Dich } \\
\text { (1: men 2: woman) }\end{array}$ \\
\hline Smith, 2012 & Moderate & 1683 & HHS & LT (36 m) & $<0.001$ & Pos, men & $\begin{array}{l}\text { Dich } \\
\text { (1: men 2: woman) }\end{array}$ \\
\hline Gandhi, 2010 & Low & 636 & $\begin{array}{l}\text { WOMAC } \\
\text { SF-36 PF }\end{array}$ & LT (39 m) & $\begin{array}{l}\text { No } \\
<0.05\end{array}$ & $\begin{array}{l}\text { No } \\
\text { Pos, woman }\end{array}$ & $\begin{array}{l}\text { Dich } \\
\text { (1: men 2: woman) }\end{array}$ \\
\hline Lavernia, 2011 & Low & 532 & WOMAC PF & LT (42 m) & $<0.001^{*}$ & Pos, woman & $\begin{array}{l}\text { Dich } \\
\text { (1: men 2: woman) }\end{array}$ \\
\hline Nilsdotter, 2003 & Low & 211 & WOMAC PF & LT (66 m) & 0.37 & No & $\begin{array}{l}\text { Dich } \\
\text { (1: men 2: woman) }\end{array}$ \\
\hline
\end{tabular}

All significant results are bold; studies that used change in function as outcome are marked with *.

BMI, body mass index; Dich, dichotomous; FU, follow-up; HHS, Harris Hip Score; LEFS, Lower Extremity Functional Scale; LT, long-term; N of pts, number of patients; NA, not applicable; Neg, negative; OHS, Oxford Hip Score; Pos, positive; SF-36 PF, Short Form 36 physical function; ST, short-term; THA, total hip arthroplasty; WOMAC, Western Ontario and Mcmaster Universities Osteoarthritis Index. 
three studies reported being female as a predictor of better functional outcome. ${ }^{21} 28$ This demonstrates inconsistent evidence for an association between gender and functional outcome after THA.

\section{Preoperative physical function}

Seventeen studies evaluated preoperative physical function as a possible predictor of functional outcome after THA $^{17} 23$ 25-29 32 34-37 39-43 (table 6). A total of 9689 patients were included in all studies that evaluated preoperative physical function, with a mean follow-up time of 16 months. The applied levels of measurement of preoperative physical function were continuous, dichotomous or categorical.

The WOMAC score ${ }^{14}$ was the measurement tool most used to determine the preoperative physical func-

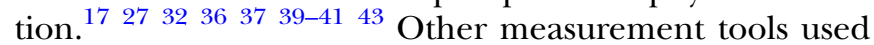
were the HHS, TUG, OHS, SF-36, SF-12 and the ambulatory status.

Of the 17 studies, 16 found a statistically significant correlation between preoperative physical function and the functional outcome. Fourteen studies evaluated the short-term outcome of which 13 reported a significant association. Three studies evaluated the long-term outcome; all three found a significant association. The only study that did not report a significant association, was a study on a small patient group that used the TUG to determine the preoperative physical function. ${ }^{28}$ Studies were designated as level of evidence low (5), moderate (9) or high (3).

As more than $60 \%$ of the studies report a significant negative association, there is strong evidence of a shortterm and long-term association between the preoperative physical function and the functional outcome after THA.

\section{Comorbidity}

Thirteen studies evaluated comorbidity as a possible predictor of functional outcome after THA (table 7). A total of 9363 patients were included in all studies that evaluated comorbidity as a possible predictor, with a mean follow-up time of 23.3 months. The applied levels of measurement of preoperative status were continuous, dichotomous or categorical.

The measurement tools used to determine the functional outcome were the WOMAC score, HHS, LEFS, SF-36 and the ambulatory status. Most studies used the number of comorbidities or American Society of Anesthestiologists (ASA) grade as predictor of functional outcome. Other studies used the presence of a specific comorbidity as a predictor like cardiac disease, coronary heart disease and thromboembolism.

Of the 13 studies, 11 found a significant negative association. ${ }^{18} 21222527293032-34373942$ Seven studies evaluated the short-term outcome of which six reported a significant negative association. ${ }^{22} 23253032 \quad 34 \quad 3942$ Six studies evaluated the long-term outcome, of which five found a significant negative association. ${ }^{18}{ }^{21-23} 29$ All articles were designated as level of evidence low (2), moderate (8) or high (3).

Since more than $60 \%$ of the studies report a significant negative association, there was strong evidence of a negative association between comorbidities and shortterm and long-term functional outcomes after THA.

\section{Other predictors}

The predictors that were evaluated in five studies or less are displayed in table 8 .

Five studies evaluated mental health as a possible predictor of functional outcome after THA, with a total of 3563 patients. ${ }^{18} 23343744$ All four studies evaluating the short-term functional outcome found a significant positive association. ${ }^{23} 343744$ Both studies that evaluated the long-term outcome found a significant positive association. Since more than $60 \%$ of the studies report a significant positive association, there is strong evidence of an association between good mental health and better short-term physical function outcome after THA. Since only two studies evaluated the long-term outcome, this evidence is weak.

Two studies evaluated alcohol consumption as a predictor of functional outcome. ${ }^{18}{ }^{45}$ Neither of them found a significant result and therefore none show evidence of an association. The two studies evaluating quadriceps strength as a possible predictor ${ }^{26}{ }^{46}$ looked at the shortterm functional outcome and both found a significant association. Therefore, the evidence for an association is weak.

All three studies that evaluated educational level as a possible predictor found a significant association. ${ }^{18} 3947$ Two studies evaluated the short-term outcome and both found a significant association. ${ }^{39} 47$ One study evaluated the long-term effect and found a significant association. ${ }^{18}$ All three studies used the WOMAC score to measure the functional outcome. These results show weak evidence for a short-term association, and incomplete evidence for a long-term association.

One study reported socioeconomic status (SES) as a predictor, using the SES score as measurement tool. ${ }^{34}$ They did not find a significant result and therefore show limited evidence of an association.

The influence of having more than three allergies on the short-term functional outcome was reported in one study. ${ }^{48}$ Patients with allergies had diminished improvements on SF-36 PCS and WOMAC scores 6.5 months after THA. There was limited evidence of an association between having more than three allergies and functional outcome.

Vitamin D insufficiency as a predictor of functional outcome after THA was evaluated in one study. ${ }^{49}$ A preoperative 25-hydroxyvitamin D3 plasma level of under $30 \mathrm{ng} / \mathrm{mL}$, predicted a worse HHS 11 months postoperative. Since no other studies evaluated vitamin D insufficiency as a possible predictor, this result shows limited evidence of an association. 
Table 6 Studies reporting preoperative physical function as possible predictor of functional outcome after THA

\begin{tabular}{|c|c|c|c|c|c|c|c|}
\hline Author, year & Grade & $\mathrm{N}$ of pts & $\begin{array}{l}\text { Measurement } \\
\text { tool }\end{array}$ & $\begin{array}{l}\text { FU period } \\
\text { (months) }\end{array}$ & $\begin{array}{l}\text { Significance } \\
\text { Level ( } p \text { value) }\end{array}$ & Association & Predictor level of measurement \\
\hline Quintana, 2009 & Moderate & 788 & $\begin{array}{l}\text { WOMAC PF } \\
\text { SF-36 PF }\end{array}$ & ST $(6 \mathrm{~m})$ & $<0.001$ & Yes & Cont (WOMAC+SF-36) \\
\hline Slaven, 2012 & Low & 40 & TUG & ST (6 m) & NA & No & $\begin{array}{l}\text { Dich } \\
\text { (successful/unsuccessful) }\end{array}$ \\
\hline Mahomed, 2002 & Moderate & 103 & $\begin{array}{l}\text { WOMAC PF+P } \\
\text { SF-36 PF }\end{array}$ & ST $(6 \mathrm{~m})$ & $\begin{array}{l}<0.05 \\
<0.05\end{array}$ & Yes & Cont (WOMAC+SF-36) \\
\hline Hamilton, 2012 & Low & 1410 & $\begin{array}{l}\text { OHS } \\
\text { SF-12 }\end{array}$ & $\begin{array}{l}\text { ST }(6 \mathrm{~m}) \\
\text { ST }(12 \mathrm{~m})\end{array}$ & Yes & Yes & Cont $(\mathrm{OHS})$ \\
\hline Nankaku, 2013 & Moderate & 204 & Ambulatory status ST (6 m) & NA & Yes & Dich (TUG score 10) & \\
\hline Vogl, 2014 & Low & 281 & WOMAC & ST $(6 \mathrm{~m})$ & NA & Yes & Cont (WOMAC) \\
\hline Bergschmidt, 2010 & Moderate & 100 & $\begin{array}{l}\text { WOMAC } \\
\text { SF-36 }\end{array}$ & ST $(12 \mathrm{~m})$ & $\begin{array}{l}<0.022 \\
0.003\end{array}$ & Yes & $\begin{array}{l}\text { Cat (3) } \\
\text { 1: HHS<48 2: HHS 48-59 3: HHS>59 }\end{array}$ \\
\hline Clement, 2010 & High & 1312 & $\begin{array}{l}\text { OHS } \\
\text { SF-12 }\end{array}$ & ST (12 m) & $0.001^{*}$ & Yes & Cont $(\mathrm{OHS})$ \\
\hline Johansson, 2010 & Moderate & 75 & $\begin{array}{l}\text { HHS } \\
\text { WOMAC } \\
\text { SF-36 }\end{array}$ & ST $(12 \mathrm{~m})$ & $\begin{array}{l}? 0.006 \\
<0.001 \\
? 0.005\end{array}$ & $\begin{array}{l}\text { Yes } \\
\text { Yes } \\
\text { Yes }\end{array}$ & $\begin{array}{l}\text { Cat (3) } \\
\text { 1: HHS<45 2: HHS 45-55 3: HHS>55 }\end{array}$ \\
\hline Nilsdotter, 2002 & Moderate & 148 & $\begin{array}{l}\text { WOMAC } \\
\text { SF-36 }\end{array}$ & ST (12 m) & $<0.0001$ & Yes & $\begin{array}{l}\text { Dich } \\
\text { Low quartile vs high quartile WOMAC }\end{array}$ \\
\hline Dowsey, 2014 & High & 835 & HHS & ST $(12 \mathrm{~m})$ & $<0.0001$ & Yes & Cont $(\mathrm{HHS})$ \\
\hline Wang, 2010 & Moderate & 97 & WOMAC & ST $(16.8 \mathrm{~m})$ & 0.0001 & Yes & Cont (WOMAC PF) \\
\hline Moran, 2005 & Moderate & 749 & $\mathrm{HHS}$ & ST $(18 \mathrm{~m})$ & NA & Yes & Cont \\
\hline Fortin, 2002 & Low & 222 & $\begin{array}{l}\text { WOMAC } \\
\text { SF-36 }\end{array}$ & ST (24 m) & $\begin{array}{l}\text { NA } \\
\text { NA }\end{array}$ & $\begin{array}{l}\text { Yes } \\
\text { Yes }\end{array}$ & Dig (1: high WOMAC 2: Iow WOMAC) \\
\hline Smith, 2012 & Moderate & 1683 & $\mathrm{HHS}$ & LT (36 m) & $<0.001$ & Yes & Cont (HHS) \\
\hline Nilsdotter, 2003 & Low & 211 & WOMAC PF & LT $(42 \mathrm{~m})$ & 0.007 & Yes & $\begin{array}{l}\text { Dich } \\
\text { Low quartile vs high quartile SF-36 PF }\end{array}$ \\
\hline Judge, 2013 & High & 1431 & $\mathrm{OHS}$ & LT $(60 \mathrm{~m})$ & $<0.001$ & Yes & Cont (OHS) \\
\hline
\end{tabular}


Table 7 Studies reporting comorbidity status as possible predictor of functional outcome after THA

\begin{tabular}{|c|c|c|c|c|c|c|c|}
\hline Author, year & Grade & $\mathrm{N}$ of pts & $\begin{array}{l}\text { Measurement } \\
\text { tool }\end{array}$ & $\begin{array}{l}\text { FU period } \\
\text { (months) }\end{array}$ & $\begin{array}{l}\text { Significance } \\
\text { level ( } p \text { value) }\end{array}$ & Association & Predictor level of measurement \\
\hline Quintana, 2009 & Moderate & 788 & $\begin{array}{l}\text { WOMAC PF } \\
\text { SF-36 PF }\end{array}$ & ST $(6 \mathrm{~m})$ & $\begin{array}{l}\text { NA } \\
\text { NA }\end{array}$ & No & $\begin{array}{l}\text { Cat }(3) \\
1: 0 \text { comorbidities } 2: 1-2 \text { comorbidities } 3:>2 \text { comorbidities }\end{array}$ \\
\hline Mahomed, 2002 & Moderate & 103 & WOMAC PF+P & $\mathrm{ST}(6 \mathrm{~m})$ & $<0.05$ & Neg & $\begin{array}{l}\text { Cont } \\
\text { (number of comorbidities) }\end{array}$ \\
\hline Moran, 2005 & Moderate & 749 & $\mathrm{HHS}$ & $\begin{array}{l}\text { ST }(6 \mathrm{~m}) \\
\text { ST }(18 \mathrm{~m})\end{array}$ & NA & Neg & $\begin{array}{l}\text { Dich } \\
\text { (presence of coronary heart disease and } \\
\text { previous thromoembolism) }\end{array}$ \\
\hline Stevens, 2012 & Moderate & 653 & WOMAC & ST (12 m) & 0.01 & Neg & $\begin{array}{l}\text { Cat }(3) \\
1: 0 \text { comorbidities } 2: 1-2 \text { comorbidities } 3:>2 \text { comorbidities }\end{array}$ \\
\hline Clement, 2010 & High & 1312 & $\begin{array}{l}\text { OHS } \\
\text { SF-12 }\end{array}$ & ST (12 m) & 0.01 & Neg & $\begin{array}{l}\text { Cont } \\
\text { (number of comorbidities) }\end{array}$ \\
\hline Dowsey, 2014 & High & 835 & HHS & $\mathrm{ST}(12 \mathrm{~m})$ & 0.0001 & Neg & $\begin{array}{l}\text { Cont } \\
\text { (age-adjusted } \mathrm{CCl} \text { ) }\end{array}$ \\
\hline Wang, 2010 & Moderate & 97 & WOMAC & ST (16.8 m) & 0.0246 & Neg & $\begin{array}{l}\text { Dich } \\
\text { (1: >0 comorbidities } 2: 0 \text { comorbidities })\end{array}$ \\
\hline Jones, 2012 & Moderate & 231 & WOMAC & LT (36 m) & 0.012 & Neg & $\begin{array}{l}\text { Dich } \\
(1 ; 0 \text { cardiac diseases } \\
2:>0 \text { cardiac diseases })\end{array}$ \\
\hline Bischoff, 2004 & Moderate & 922 & WOMAC PF & LT (36 m) & NA & $\mathrm{Neg}$ & $\begin{array}{l}\text { Dich } \\
(1 ;>2 \text { chronic diseases } \\
\text { 2. } 0-1 \text { chronic diseases })\end{array}$ \\
\hline Smith, 2012 & Moderate & 1683 & HHS & LT $(36$ m) & $<0.001$ & Neg & $\begin{array}{l}\text { Cont } \\
\text { (ASA grade) }\end{array}$ \\
\hline Gandhi, 2010 & Low & 636 & $\begin{array}{l}\text { WOMAC } \\
\text { SF-36 PF }\end{array}$ & LT $(39$ m) & $<0.05$ & Neg & $\begin{array}{l}\text { (number of comorbidities) } \\
\text { (numt }\end{array}$ \\
\hline Nilsdotter, 2003 & Low & 211 & WOMAC PF & LT (42 m) & 0.08 & No & $\begin{array}{l}\text { Dich } \\
\text { (1: >1 comorbidities } 2: 0-1 \text { comorbidities) }\end{array}$ \\
\hline Judge, 2013 & High & 1431 & $\mathrm{OHS}$ & LT $(60 \mathrm{~m})$ & 0.001 & Neg & $\begin{array}{l}\text { Cont } \\
\text { (number of comorbidities) }\end{array}$ \\
\hline
\end{tabular}

All significant results are bold.

BMI, body mass index; Cat, categorical; CCI, Charlson comorbidity index; Cont, continuous; Dich, dichotomous; FU, follow-up; HHS, Harris Hip Score; LEFS, Lower Extremity Functional Scale;

LT, long-term; N of pts, number of patients; NA, not applicable; Neg, negative; OHS, Oxford Hip Score; Pos, positive; SF-36 PF, Short Form 36 physical function; ST, short-term; THA, total hip

arthroplasty; WOMAC, Western Ontario and Mcmaster Universities Osteoarthritis Index. 
Table 8 All predictors that are evaluated in five studies or less

\begin{tabular}{|c|c|c|c|c|c|c|c|c|}
\hline Predictor & Author, year & Grade & $\mathrm{N}$ of pts & $\begin{array}{l}\text { Measurement } \\
\text { tool }\end{array}$ & $\begin{array}{l}\text { FU-period } \\
\text { (months) }\end{array}$ & $\begin{array}{l}\text { Significance } \\
\text { level ( } p \text { value) }\end{array}$ & Association & Predictor level of measurement \\
\hline \multirow[t]{6}{*}{ Mental health } & $\begin{array}{l}\text { Badura-Brzoza, } \\
2009\end{array}$ & Moderate & 102 & SF-36 PCS & ST $(6 \mathrm{~m})$ & 0.005 & Neg & $\begin{array}{l}\text { Cont } \\
\text { (anxiety as a trait) }\end{array}$ \\
\hline & Quintana, 2009 & Moderate & 788 & SF-36 PF & ST $(6 \mathrm{~m})$ & $<0.001$ & Yes & Cont \\
\hline & & & & WOMAC P & ST $(24 \mathrm{~m})$ & 0.002 & & (SF-36 MH score) \\
\hline & Dowsey, 2014 & High & 835 & HSS & ST $(12 \mathrm{~m})$ & $<0.0001$ & Yes & $\begin{array}{l}\text { Cont } \\
\text { (SF-12 MH score) }\end{array}$ \\
\hline & Bischoff, 2004 & Moderate & 922 & WOMAC PF & LT $(36$ m) & NA & Yes & $\begin{array}{l}\text { Dich } \\
(1:>60 \text { pts on the SF-36 MH score } \\
\text { 2: } ? 60 \text { pts on SF-36 MH score) }\end{array}$ \\
\hline & Judge, 2013 & High & 916 & $\mathrm{OHS}$ & $\begin{array}{l}\text { ST }(12 \mathrm{~m}) \\
\text { LT }(60 \mathrm{~m})\end{array}$ & 0.045 & Yes & $\begin{array}{l}\text { Cont } \\
\text { (SF-36 MH score) }\end{array}$ \\
\hline \multirow[t]{2}{*}{$\begin{array}{l}\text { Alcohol } \\
\text { consumption }\end{array}$} & Bischoff, 2004 & Moderate & 914 & WOMAC PF & LT $(36 \mathrm{~m})$ & NA & No & $\begin{array}{l}\text { Dich } \\
(1:>1 \text { alcoholic drinks per day } \\
\text { 2: } 0-1 \text { alcoholic drinks per day })\end{array}$ \\
\hline & Lavernia, 2014 & Low & 191 & WOMAC & LT $(72 \mathrm{~m})$ & NA & No & $\begin{array}{l}\text { Cat (3) } \\
\text { (1: non-drinkers 2: occasional drinkers } \\
\text { 3: moderate drinkers) }\end{array}$ \\
\hline \multirow[t]{3}{*}{$\begin{array}{l}\text { Quadriceps } \\
\text { strength }\end{array}$} & Holstege, 2011 & Moderate & 55 & WOMAC PF & ST (3 m) & 0.004 & Pos & $\begin{array}{l}\text { Cont } \\
\text { (knee extensor strength) }\end{array}$ \\
\hline & Nankaku, 2013 & Moderate & 204 & $\begin{array}{l}\text { Ambulatory } \\
\text { status }\end{array}$ & ST (6 m) & NA & Pos & Dich \\
\hline & & & & & & & & $\begin{array}{l}\text { (1: >1.25 N m/kg 2: ?1.25 m/kg } \\
\text { knee extensor strength) }\end{array}$ \\
\hline \multirow[t]{3}{*}{ Education } & Schafer, 2010 & Low & 1007 & WOMAC & ST $(6 \mathrm{~m})$ & NA & Pos & $\begin{array}{l}\text { Dich } \\
(1 ;>12 \text { years school } 2:<9 \text { years school })\end{array}$ \\
\hline & Mahomed, 2002 & Moderate & 103 & WOMAC PF+P & ST (6 m) & 0.007 & Pos & $\begin{array}{l}\text { Cont } \\
\text { (level of education) }\end{array}$ \\
\hline & Bischoff, 2004 & Moderate & 922 & WOMAC PF & LT $(36 \mathrm{~m})$ & NA & Pos & $\begin{array}{l}\text { Dich } \\
\text { (1: college education } 2 \text { : less } \\
\text { than college education) }\end{array}$ \\
\hline $\begin{array}{l}\text { Socioeco } \\
\text { nomic status }\end{array}$ & Dowsey, 2014 & High & 835 & HHS & LT (12 m) & 0.63 & No & $\begin{array}{l}\text { Cont } \\
\text { (SES score) }\end{array}$ \\
\hline Allergies & Graves, 2014 & Moderate & 459 & $\begin{array}{l}\text { WOMAC PF } \\
\text { SF-36 PCS }\end{array}$ & ST $(6.5 \mathrm{~m})$ & $\begin{array}{l}0.04 \\
0.0002\end{array}$ & $\mathrm{Neg}$ & $\begin{array}{l}\text { Dich } \\
\text { (>3 allergies) }\end{array}$ \\
\hline $\begin{array}{l}\text { Vitamin D } \\
\text { insufficiency }\end{array}$ & Lavernia, 2013 & Moderate & 60 & $\begin{array}{l}\text { HHS } \\
\text { WOMAC }\end{array}$ & ST $(11 \mathrm{~m})$ & $\begin{array}{l}0.002 \\
0.478\end{array}$ & Neg & $\begin{array}{l}\text { Dich (25-hydroxyvitamin D3) } \\
(1 ;>30 \mathrm{ng} / \mathrm{mL} 2:<30 \mathrm{ng} / \mathrm{mL})\end{array}$ \\
\hline
\end{tabular}

All significant results are bold.

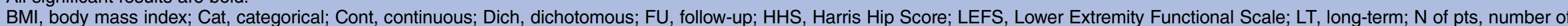
patients; NA, not applicable; Neg, negative; OHS, Oxford Hip Score; PCS, physical component summary scale; Pos, positive; SF-36 PF, Short Form 36 physical function; ST, short-term; WOMAC, Western Ontario and McMaster Universities Osteoarthritis Index. 


\section{DISCUSSION}

In this systematic literature review, we sought to provide a clear overview of a range of patient-related predictors of functional outcome after THA.

\section{Key findings}

Our review found strong evidence of an association of BMI, age, comorbidity, preoperative physical function and mental health with functional outcome after THA. Weak evidence was found for the predictors like quadriceps strength and education. Inconsistent evidence was found for the predictors like gender and SES. Limited evidence was found for the predictors like alcohol consumption, vitamin D insufficiency and allergies.

In our review, 13 studies found a significant negative association between BMI and functional outcome after THA. A prior review of Young et al found the same significant negative association. Although the review of Young et al and our current review come to the same conclusion, the clinical impact of this outcome is still questionable. A large study by Judge $e t a p^{33}$ showed a small significant correlation between high BMI and worse functional outcome, but concluded that the total improvement in function outweighs the small lack of improvement caused by high BMI.

Although our review shows strong evidence of an association between BMI and functional outcome, different classifications of high BMI were used. Owing to these different classifications, it is difficult to define a specific BMI that predicts who will do well after THA. We could not conduct a meta-analysis since different classifications of BMI were used and there was heterogeneity in outcome instruments. Therefore, future research on the impact of BMI should use clearly defined outcomes that are consistent across studies.

In our review, 8 of the 14 studies found an association between higher age and poorer functional outcome; therefore, age is an important factor predicting functional outcome. Some articles used a linear regression analysis for age. When looking at age, it is interesting to see the effect of high age, and also of low age. Therefore, linear regression analysis might not be the best statistical analysis with variables as age or BMI. There is no consensus among studies about what specific age limit is recommended for THA. This current review shows inconclusive evidence of an association between gender and functional outcome because 6 out of 14 studies found a statistically significant result.

Three studies reported being female led to a better functional outcome. ${ }^{21} 2838$ The other four significant articles found the opposite result where being male had a positive association with functional outcome after THA. ${ }^{29} 303237$ The results are contradictory and the differences may be attributable to confounding factors.

Preoperative physical function was found to be a strong predictor of long-term functional outcome. With the exception of one study reporting the TUG test as an outcome, better preoperative physical function was consistently associated with better long-term physical function. ${ }^{28}$ This might be due to the use of TUG score as measurement tool. ${ }^{28}$ The WOMAC score was the measurement tool most used to define the preoperative status (nine times). ${ }^{172732363739-4143}$ Other preoperative measurement tools that were good predictors of functional outcome were the HHS, OHS, SF-12 PF, SF-36 $\mathrm{PF}$ and the ambulatory status.

Of the 13 studies that evaluated comorbidity as a possible predictor of functional outcome, 11 found a significant negative association. ${ }^{18} \quad 21-23 \quad 25 \quad 29 \quad 30 \quad 32 \quad 34 \quad 37 \quad 3942$ Comorbidity can be measured in several ways, for example, the number of comorbidities, the presence of a specific comorbidity, the Charlson index ${ }^{50}$ and the Elixhauser comorbidity measure. ${ }^{51}$ Comorbidities can affect the true functional outcome after THA but can also affect the score on the measurement tool. For example, if a patient is unable to walk to the grocery store after a THA due to a lung disease, his functional outcome score will be lower despite a possible good functioning total hip. Except for one article, all studies found a significant negative effect. Therefore, having comorbidities can be seen as a predictor of negative functional outcome.

All five studies that evaluated mental health as a predictor of functional outcome found a statistically significant positive association. Four of these studies used SF-36 $\mathrm{MH}^{52}$ as the measurement tool to measure mental health. ${ }^{18} 233437$ These results show strong evidence of a positive association between mental health and shortterm functional outcome after THA. The two studies reporting quadriceps strength as a predictor had both small sample sizes which can affect the external validity of the studies. ${ }^{26}{ }^{46}$ Therefore, this evidence is weak and more research must be carried out on the effect of quadriceps strength.

Three studies evaluated education as predictor of functional outcome. Mahomed et $a l^{39}$ and Bischoff et $a l^{18}$ used the level of school education as a predictor, and Schäfer et $a l^{47}$ used years of education as a predictor. Since education is in part a surrogate of SES, this might also indicate that low SES is a factor associated with poor functional outcome. Dowsey et $a l^{34}$ however did not find a correlation between SES and functional outcome. Future research is needed on various components of SES to specify the impact on functional outcome. As only one study evaluated each of the allergies $^{48}$ and vitamin $\mathrm{D}$ insufficiency ${ }^{49}$ as possible predictors of functional outcome, no conclusions can be drawn.

\section{Previous systematic reviews}

The previous systematic review of Young et al concluded that important research remained to be done to examine the magnitude and interaction of patient factors on the outcome of THA. ${ }^{6}$ The review of Young et al used only one database (MEDLINE) and is more than 15 years old. Young et al also looked at implant survivorship. In our systematic review, we used multiple 
databases (Web of Science, CINAHL; EMBASE and PubMed) and reported only patient-related predictors evaluated in the literature.

\section{Strengths and limitations}

We included a range of patient-related predictors and did not limit ourselves to the most common predictors. This led to a broad overview of predictors evaluated. The reason we could not apply a meta-analysis is because of the heterogeneity across studies regarding measurement tools, predictors and duration of follow-up. Not all studies used in this review adjusted their outcomes for potential confounders. Therefore, some outcomes may be due to confounding factors. A limitation of our review is that we looked at functional outcome without including pain. Some patients will not see an improvement in their function after THA, but will lose the hip-related pain. For this reason especially people with a high BMI and older age can benefit from THA, without improving the function of the hip. Some predictors such as quadriceps strength, education, SES and alcohol consumption are reported only a few times and therefore conclusions cannot be reached. More research in large data sets is needed to draw definitive conclusions on these predictors.

\section{Implications for practice}

Our review provides a clear overview of the current literature on the predictors for physical functioning after THA. Orthopaedic surgeons and general practitioners can use this information to predict the improvement in physical functioning of their patients and it enables them to provide patient-specific advice on THA.

\section{Implications for future research}

In the future, we suggest studies that evaluate possible predictors of functional outcome after THA to use similar measurement tools, outcomes and durations of follow-up. In that way a meta-analysis can be applied and the influence of these factors can be specified.

\section{CONCLUSION}

This review shows that several patient-related characteristics can predict the functional outcome after THA. It shows strong evidence of an association between BMI, age, comorbidity, preoperative physical function and mental health with functional outcome after THA. Weak evidence suggested that quadriceps strength and education were predictive of functional outcomes after THA. Inconsistent evidence was found for the predictors like gender and SES. Alcohol consumption, vitamin D insufficiency and allergies showed limited evidence predicting functional outcome after THA. Understanding predictors will help orthopaedic surgeons and general practitioners predict the outcomes in physical functioning after THA; they can use this information to provide patient-specific advice and target care for patients with
THA. Further well-conducted cohort studies are necessary to confirm these findings.

Acknowledgements The authors would like to thank the medical librarian Bert Berenschot at OLVG for his help with the literature search.

Contributors LDB, LWAHVB, VABS, TP, SS and RWP made substantial contributions to the conception and design, or acquisition of data, or analysis and interpretation of data. LDB, LWAHVB, VABS, SS and RWP have been involved in drafting the manuscript or revising it critically for important intellectual content. All authors read and approved the final manuscript.

Competing interests LWAHVB beers report grants from Link/Lima, grants from Stryker, outside the submitted work. SS reports employment/salary from McMaster University, employment/salary from Global Research Solutions, outside the submitted work. VABS reports grants from Link/Lima, grants from Stryker, grants from NuVasive, grants from Zonmw, grants from Achmea and grants from Tornier, outside the submitted work. RWP reports grants from Link/Lima, grants from Stryker, grants from NuVasive, grants from Zonmw, grants from Achmea and grants from Tornier, outside the submitted work.

Provenance and peer review Not commissioned; externally peer reviewed.

Data sharing statement No additional data are available.

Open Access This is an Open Access article distributed in accordance with the Creative Commons Attribution Non Commercial (CC BY-NC 4.0) license, which permits others to distribute, remix, adapt, build upon this work noncommercially, and license their derivative works on different terms, provided the original work is properly cited and the use is non-commercial. See: http:// creativecommons.org/licenses/by-nc/4.0/

\section{REFERENCES}

1. Bernstein J, Derman P. Dramatic increase in total knee replacement utilization rates cannot be fully explained by a disproportionate increase among younger patients. Orthopedics 2014;37:e656-9.

2. Judge A, Cooper C, Williams S, et al. Patient-reported outcomes one year after primary hip replacement in a European Collaborative Cohort. Arthritis Care Res (Hoboken) 2010;62:480-8.

3. Singh JA, Lewallen DG. Predictors of activity limitation and dependence on walking aids after primary total hip arthroplasty. J Am Geriatr Soc 2010;58:2387-93.

4. Williams O, Fitzpatrick R, Hajat S, et al. Mortality, morbidity, and 1-year outcomes of primary elective total hip arthroplasty. $J$ Arthroplasty 2002;17:165-71.

5. MacWilliam $\mathrm{CH}$, Yood MU, Verner JJ, et al. Patient-related risk factors that predict poor outcome after total hip replacement. Health Serv Res 1996;31:623-38.

6. Young NL, Cheah D, Waddell JP, et al. Patient characteristics that affect the outcome of total hip arthroplasty: a review. Can J Surg 1998;41:188-95.

7. Nilsdotter A, Bremander A. Measures of hip function and symptoms: Harris Hip Score (HHS), Hip Disability and Osteoarthritis Outcome Score (HOOS), Oxford Hip Score (OHS), Lequesne Index of Severity for Osteoarthritis of the Hip (LISOH), and American Academy of Orthopedic Surgeons (AAOS) Hip and Knee Questionnaire. Arthritis Care Res (Hoboken) 2011;63(Suppl 11):S200-7.

8. Gosens T, Hoefnagels NHM, de Vet RCW, et al. The "Oxford Heup Score": the translation and validation of a questionnaire into Dutch to evaluate the results of total hip arthroplasty. Acta Orthop 2005;76:204-11.

9. Dawson J, Fitzpatrick R, Carr A, et al. Questionnaire on the perceptions of patients about total hip replacement. J Bone Joint Surg Br 1996;78:185-90.

10. Brazier JE, Harper R, Jones NM, et al. Validating the SF-36 health survey questionnaire: new outcome measure for primary care. BMJ 1992;305:160-4.

11. Binkley JM, Stratford PW, Lott SA, et al. The Lower Extremity Functional Scale (LEFS): scale development, measurement properties, and clinical application. North American Orthopaedic Rehabilitation Research Network. Phys Ther 1999;79:371-83.

12. Podsiadlo D, Richardson S. The timed "Up \& Go": a test of basic functional mobility for frail elderly persons. J Am Geriatr Soc 1991;39:142-8.

13. Mathias S, Nayak US, Isaacs B. Balance in elderly patients: the "get-up and go" test. Arch Phys Med Rehabil 1986;67:387-9. 
14. Bellamy N, Buchanan WW, Goldsmith $\mathrm{CH}$, et al. Validation study of WOMAC: a health status instrument for measuring clinically important patient relevant outcomes to antirheumatic drug therapy in patients with osteoarthritis of the hip or knee. J Rheumatol 1988; 15:1833-40.

15. Singh AS, Mulder C, Twisk JW, et al. Tracking of childhood overweight into adulthood: a systematic review of the literature. Obes Rev 2008;9:474-88.

16. Moher D, Liberati A, Tetzlaff J, et al. Preferred reporting items for systematic reviews and meta-analyses: the PRISMA statement. Ann Intern Med 2009;151:264-9.

17. Bergschmidt $\mathrm{P}$, Bader R, Finze S, et al. Impact of preoperative variables on the functional and radiological outcome of an uncemented femoral stem: a prospective two-year follow-up. Hip Int 2010;20:187-97.

18. Bischoff-Ferrari HA, Lingard EA, Losina E, et a/Psychosocial and geriatric correlates of functional status after total hip replacement. Arthritis Rheum 2004;51:829-35.

19. Davis AM, Wood AM, Keenan AC, et al. Does body mass index affect clinical outcome post-operatively and at five years after primary unilateral total hip replacement performed for osteoarthritis? A multivariate analysis of prospective data. J Bone Joint Surg $\mathrm{Br}$ 2011;93:1178-82

20. Dowsey MM, Liew D, Stoney JD, et al. The impact of obesity on weight change and outcomes at 12 months in patients undergoing total hip arthroplasty. Med J Aust 2010;193:17-21.

21. Gandhi R, Dhotar H, Davey JR, et al. Predicting the longer-term outcomes of total hip replacement. $J$ Rheumatol 2010;37:2573-7.

22. Jones CA, Cox V, Jhangri GS, et al. Delineating the impact of obesity and its relationship on recovery after total joint arthroplasties. Osteoarthr Cartil 2012;20:511-18.

23. Judge A, Arden NK, Batra RN, et al. The association of patient characteristics and surgical variables on symptoms of pain and function over 5 years following primary hip-replacement surgery: a prospective cohort study. BMJ Open 2013;3:pii: e002453.

24. Kessler S, Käfer W. Overweight and obesity: two predictors for worse early outcome in total hip replacement? Obesity (Silver Spring) 2007;15:2840-5.

25. Moran M, Walmsley P, Gray A, et al. Does body mass index affect the early outcome of primary total hip arthroplasty? J Arthroplasty 2005;20:866-9

26. Nankaku M, Tsuboyama T, Akiyama $\mathrm{H}$, et al. Preoperative prediction of ambulatory status at 6 months after total hip arthroplasty. Phys Ther 2013;93:88-93.

27. Nilsdotter AK, Petersson IF, Roos EM, et al. Predictors of patient relevant outcome after total hip replacement for osteoarthritis: a prospective study. Ann Rheum Dis 2003;62:923-30.

28. Slaven EJ. Prediction of functional outcome at six months following total hip arthroplasty. Phys Ther 2012;92:1386-94.

29. Smith GH, Johnson S, Ballantyne JA, et al. Predictors of excellent early outcome after total hip arthroplasty. J Orthop Surg Res 2012;7:13.

30. Stevens M, Paans N, Wagenmakers R, et al. The influence of overweight/obesity on patient-perceived physical functioning and health-related quality of life after primary total hip arthroplasty. Obes Surg 2012:22:523-9.

31. Aranda Villalobos $P$, Navarro-Espigares JL, Hernández-Torres E, et al. Body mass index as predictor of health-related quality-of-life changes after total hip arthroplasty: a cross-over study. $J$ Arthroplasty 2013;28:666-70.

32. Wang W, Morrison TA, Geller JA, et al. Predicting short-term outcome of primary total hip arthroplasty: a prospective multivariate regression analysis of 12 independent factors. J Arthroplasty 2010;25:858-64.
33. Judge A, Batra RN, Thomas GE, et al. Body mass index is not a clinically meaningful predictor of patient reported outcomes of primary hip replacement surgery: prospective cohort study. Osteoarthr Cartil 2014:22:431-9.

34. Dowsey MM, Nikpour M, Choong PFM. Outcomes following large joint arthroplasty: does socio-economic status matter? BMC Musculoskelet Disord 2014;15:148.

35. Hamilton D, Henderson GR, Gaston P, et al. Comparative outcomes of total hip and knee arthroplasty: a prospective cohort study. Postgrad Med J 2012;88:627-31.

36. Nilsdotter AK, Lohmander LS. Age and waiting time as predictors of outcome after total hip replacement for osteoarthritis. Rheumatology (Oxford) 2002;41:1261-7.

37. Quintana JM, Escobar A, Aguirre U, et al. Predictors of health-related quality-of-life change after total hip arthroplasty. Clin Orthop Relat Res 2009;467:2886-94.

38. Lavernia CJ, Alcerro JC, Contreras JS, et al. Patient perceived outcomes after primary hip arthroplasty: does gender matter? Clin Orthop Relat Res 2011;469:348-54.

39. Mahomed NN, Liang MH, Cook EF, et al. The importance of patient expectations in predicting functional outcomes after total joint arthroplasty. J Rheumatol 2002;29:1273-9.

40. Johansson HR, Bergschmidt P, Skripitz R, et al. Impact of preoperative function on early postoperative outcome after total hip arthroplasty. J Orthop Surg (Hong Kong) 2010;18:6-10.

41. Fortin PR, Penrod JR, Clarke AE, et a/Timing of total joint replacement affects clinical outcomes among patients with osteoarthritis of the hip or knee. Arthritis Rheum 2002:46:3327-30.

42. Clement ND, Muzammil A, Macdonald D, et al. Socioeconomic status affects the early outcome of total hip replacement. $J$ Bone Joint Surg Br 2011;93:464-9.

43. Vogl M, Wilkesmann R, Lausmann C, et al. The impact of preoperative patient characteristics on health states after total hip replacement and related satisfaction thresholds: a cohort study. Health Qual Life Outcomes 2014;12:108.

44. Badura-Brzoza K, Zajac P, Kasperska-Zajac A, et al. Anxiety and depression and their influence on the quality of life after total hip replacement: preliminary report. Int $J$ Psychiatry Clin Pract 2008;12:280-4.

45. Lavernia CJ, Villa JM, Contreras JS. Alcohol use in elective total hip arthroplasty: risk or benefit? Clin Orthop Relat Res 2013;471:504-9.

46. Holstege MS, Lindeboom R, Lucas C. Preoperative quadriceps strength as a predictor for short-term functional outcome after total hip replacement. Arch Phys Med Rehabil 2011;92:236-41.

47. Schäfer T, Krummenauer F, Mettelsiefen J, et al. Social, educational, and occupational predictors of total hip replacement outcome. Osteoarthr Cartil 2010;18:1036-42.

48. Graves CM, Otero JE, Gao Y, et al. Patient reported allergies are a risk factor for poor outcomes in total hip and knee arthroplasty. $J$ Arthroplasty 2014:29(Suppl 9):147-9.

49. Lavernia CJ, Villa JM, lacobelli DA, et al. Vitamin D insufficiency in patients with THA: prevalence and effects on outcome. Clin Orthop Relat Res 2014:472:681-6.

50. Charlson ME, Pompei P, Ales KL, et al. A new method of classifying prognostic comorbidity in longitudinal studies: development and validation. J Chronic Dis 1987:40:373-83.

51. van Walraven $C$, Austin PC, Jennings A, et al. A modification of the Elixhauser comorbidity measures into a point system for hospital death using administrative data. Med Care 2009;47:626-33.

52. McHorney CA, Ware JEJ, Raczek AE. The MOS 36-item Short-Form Health Survey (SF-36): II. Psychometric and clinical tests of validity in measuring physical and mental health constructs. Med Care 1993;31:247-63. 